\title{
New markers of early cardiovascular risk in multiple sclerosis patients: Oxidized-LDL correlates with clinical staging
}

\author{
Filipe Palavra ${ }^{\mathrm{a}, \mathrm{b}, *}$, Daniela Marado ${ }^{\mathrm{c}}$, Filipa Mascarenhas-Melo ${ }^{\mathrm{b}}$, José Sereno $^{\mathrm{b}}$, Edite Teixeira-Lemos ${ }^{\mathrm{d}}$, \\ Carla Cecília Nunes ${ }^{\mathrm{a}}$, Grilo Gonçalves ${ }^{\mathrm{a}}$, Frederico Teixeira ${ }^{\mathrm{b}}$ and Flávio Reis ${ }^{\mathrm{b}}$ \\ ${ }^{a}$ Neurology Department, General Hospital, University and Hospital Centre of Coimbra, Coimbra, Portugal \\ ${ }^{\mathrm{b}}$ Laboratory of Pharmacology and Experimental Therapeutics, IBILI, Medicine Faculty, Coimbra University, \\ Coimbra, Portugal \\ ${ }^{\mathrm{c}}$ Internal Medicine Department, General Hospital, University and Hospital Centre of Coimbra, Coimbra, Portugal \\ ${ }^{\mathrm{d}}$ ESAV and Educational Technologies and Health Study Center, Polytechnic Institute of Viseu, Campus Politécnico, \\ Viseu, Portugal
}

\begin{abstract}
.
OBJECTIVES: This study aimed to characterize a population of multiple sclerosis (MS) patients in terms of traditional and new cardiovascular risk factors and assess their putative correlation with clinical disease activity (evaluated by the Expanded Disability Status Scale [EDSS]).

METHODS: Thirty relapsing MS patients and 66 subjects, matched by age and sex, were enrolled in this cross-sectional study. For each subject, anthropometric data were collected and classical biochemical (including lipid profile, glucose and $\mathrm{C}$ reactive protein [CRP] levels) and novel markers (paraoxonase 1 [PON1] enzyme activity and contents of high-density lipoprotein [HDL] cholesterol, oxidized low-density lipoprotein [Ox-LDL], tumor necrosis factor [TNF]-alfa, vascular endothelial growth factor [VEGF] and adiponectin) were studied.

RESULTS: In patients group, 23 women and 7 men were included, aged 35.00 (28.25-40.25) years and scoring a median of 2.00 (1.50-3.13) in EDSS. Comparing with controls, the most relevant differences encountered were: increased serum triglycerides $(P<0.001)$, Ox-LDL $(P<0.001)$ as well as Ox-LDL/LDL ratio and reduced small HDL $(P=0.040)$, accompanied by a trend to increased VEGF concentration. LDL content, especially Ox-LDL, showed positive and significant correlation with EDSS ( $r=$ $0.458 ; P=0.011)$ and $\operatorname{VEGF}(r=0.453 ; P=0.014)$.

CONCLUSIONS: MS patients presented a profile of early CV risk, being Ox-LDL contents a putative good marker and having correlation with the clinical activity of the disease.
\end{abstract}

Keywords: Multiple sclerosis, early cardiovascular risk, EDSS, oxidized-LDL, small HDL, VEGF

\section{Introduction}

Multiple sclerosis (MS) is a demyelinating disease of the central nervous system (CNS), being responsi-

${ }^{*}$ Corresponding author: Filipe Palavra, Neurology Department, General Hospital, University and Hospital Centre of Coimbra, Quinta dos Vales, S. Martinho do Bispo, 3041-801 Coimbra, Portugal. Tel.: +351 239800 052; Fax: +351 239800 179; E-mail: filipepalavra@gmail.com. ble for the majority of neurological disability among young adults. According to the World Health Organization, its median prevalence is of 80 per 100.000 people in Europe [1]. The precise etiology of the disease is still unknown, although epidemiological data indicate that genetic and environmental factors are important [2]. Despite data are still scarce, studies point to an increased cardiovascular risk (CVR) in patients with $\mathrm{MS}$, at least when compared with individuals of similar age [3-5]. MS patients exhibit autonomic dysfunc- 
tion [6], increased blood pressure and tachycardia [7], ventricular disturbances [8] and impairment of the balance between vasoconstrictors and vasodilators, such as prostaglandins and nitric oxide (NO) $[9,10]$. However, there is still no clear explanation for all the aspects that may underlie these findings.

Chronic inflammation is also an important factor to be considered in the development of atherosclerosis, also contributing to raise the overall CVR, by several factors. Content and/or lipoproteins functionality may play a role in the development and progression of MS [11]. Paraoxonase 1 (PON1) is an enzyme which is connected to high-density lipoprotein (HDL), thought to be responsible for anti-atherogenic and antioxidant properties of such lipoproteins, giving also protection against oxidized low-density lipoprotein (Ox-LDL) [12]. Several studies have shown a strong relationship between their activity and the development of atherosclerosis. However, its potential involvement in injury and premature atherosclerotic disease in patients with MS remains to be clarified. Moreover, inflammatory imbalances, viewed by increased pro-inflammatory cytokines, such as TNF- $\alpha$ and/or reduced anti-inflammatory and anti-atherogenic molecules, including adiponectin, have been considered key factors for the CVR in other pathologies [13, 14] and deserve attention in MS. A similar effect has been given to the phenomenon of angiogenesis, being the vascular endothelial growth factor (VEGF) a new biomarker with increased importance $[15,16]$.

Based on this background and considering the limited information available on the role of new biomarkers for assessment of cardiometabolic risk, we aimed, with this study, to characterize a population of MS patients in terms of traditional and new CVR factors (and, comparing with controls, identify the more relevant markers in corroborating patients' higher theoretical vascular risk) and assess their putative correlation with clinical disease activity (evaluated by Kurtzke's Expanded Disability Status Scale [EDSS]) [17].

\section{Materials and methods}

\subsection{Ethics statement}

This study took place after authorization by the Ethics Committee of the University and Hospital Centre of Coimbra and upon obtaining written informed consent from the patient, by filling in a specific document, which was also approved by that Committee.
The study was performed in agreement with the Code of Ethics of the World Medical Association (Declaration of Helsinki).

\subsection{Study design}

This was a single-center, cross-sectional study. Thirty MS patients and 66 healthy volunteers, matched by age and sex, without any known neurological or cardiovascular disease (and functioning as a control group) were enrolled in the study. For each subject, anthropometric data were collected (age, sex, weight, body mass index $[\mathrm{BMI}]$, waist circumference and systolic and diastolic blood pressure), classical biochemical (lipid profile, glucose and CRP were also studied and novel cardiometabolic risk markers (PON1 activity, content of Ox-LDL, TNF- $\alpha$, VEGF and adiponectin) were determined. For MS patients, EDSS rate was also registered, at the day of study enrollment.

\subsection{Patient population}

The study population included 30 patients with relapsing-remitting MS (RRMS), consecutively enrolled from our MS Clinic, satisfying the following inclusion criteria: 1) to be aged between 18 and 55 years; 2) to be diagnosed with RRMS according to the revised McDonald criteria (2005) [18], for, at least, 6 months; 3 ) to be under stable treatment with first line immunomodulatory drugs (glatiramer acetate or interferon beta) or natalizumab for, at least, 3 months; 4) to be on fasting condition at the day of blood collection; 5) to have given the informed consent for inclusion in the study. Patients with the following conditions were excluded: 1) those who had a history of MS relapse in the last 3 months; 2) those who were submitted to corticosteroid treatment in the last 3 months; 3 ) those who have a history of autoimmune and inflammatory diseases, beyond MS; 4) those patients who had a clinical and laboratorial documentation of infection in the last 3 months. Patients were under the following treatments: interferon beta-1a $(n=10)$; interferon beta-1b $(n=8)$; glatiramer acetate $(n=7)$; natalizumab $(n=5)$; selective serotonin reuptake inhibitors $(n=8)$; tricyclic antidepressants $n=4)$; benzodiazepines $(n=4)$; voltage-dependent calcium channels inhibitors $(n=1)$; angiotensin-converting enzyme inhibitors $(n=1)$; statins $(n=1)$. 
Table 1

Anthropometric data and clinical staging of multiple sclerosis (MS) patients and age and sex-matched controls

\begin{tabular}{|c|c|c|c|}
\hline Parameters & Controls $(n=66)$ & MS patients $(n=30)$ & $P^{*}$ \\
\hline EDSS (0 to 10$)$ & - & $2.00(1.50-3.13)$ & - \\
\hline Male/Female $[\mathrm{n}(\%)]$ & $16 / 50(24 \% / 76 \%)$ & $7 / 23(23 \% / 77 \%)$ & - \\
\hline Age (years) & $35.50(26.75-43.00)$ & $35.00(28.25-40.25)$ & 0.613 \\
\hline $\mathrm{BMI}\left(\mathrm{Kg} / \mathrm{m}^{2}\right)$ & $26.48(22.11-26.59)$ & $23.75(21.98-28.43)$ & 0.931 \\
\hline Waist circumference $(\mathrm{cm})$ & $90.00(81.00-95.50)$ & $86.40(80.75-96.00)$ & 0.436 \\
\hline $\mathrm{SBP}(\mathrm{mmHg})$ & $124.00(112.00-134.00)$ & $115.00(110.00-121.00)$ & 0.011 \\
\hline $\mathrm{DBP}(\mathrm{mmHg})$ & $78.00(71.50-86.50)$ & $72.50(69.00-77.00)$ & 0.001 \\
\hline Glycemia (mmol/L) & $4.70(4.51-5.10)$ & $4.76(4.16-5.70)$ & 0.739 \\
\hline
\end{tabular}

Results are presented as median (inter quartile range). ${ }^{*}$ Mann-Whitney test. EDSS, Expanded Disability Status Scale; BMI, body mass index; SBP, systolic blood pressure; DBP, diastolic blood pressure.

\subsection{Laboratory procedures}

\subsubsection{Blood samples collection}

The blood samples were collected by venopuncture from control subjects and MS patients after an overnight fasting period, to EDTA containing tubes and to tubes without anticoagulant, in order to obtain plasma, buffy-coat and serum, and processed within $2 \mathrm{~h}$ of collection. Aliquots were immediately stored at $-80^{\circ} \mathrm{C}$ until assayed.

\subsubsection{Lipid profile}

Serum lipids and lipoproteins analysis were performed in an auto-analyser (Cobas Mira S, Roche), using commercially available kits. Serum total cholesterol (TC) and triglycerides (TGs) concentrations were determined by enzymatic colorimetric tests (CHODPAP and GPO-PAP methods, Roche, respectively). HDL-c and LDL-c levels were measured by using enzymatic colorimetric tests, after selective separation of HDL and LDL fractions (Direct HDLCholesterol and Direct LDLCholesterol, Roche). Plasma concentration of Ox-LDL was evaluated by using a standard commercial enzyme-linked immunoassay (Oxidized LDL ELISA, Mercodia, eBioscience). HDLc subpopulations were separated and quantified using a Lipoprint kit from Quantimetrix Corp. (Redondo Beach, CA, USA). The assay involves a polyacrylamide gel electrophoresis assay and a complete Lipoprint System for data acquisition and quantification of Large, Intermediary and Small subpopulations of HDL. Serum paraoxonase activity of PON1 was assessed spectrophotometrically and expressed in nmol of pnitrophenol $/ \mathrm{ml} / \mathrm{min}$. In brief, paraoxonase activity was measured by adding serum to $1 \mathrm{~mL}$ Tris $/ \mathrm{HCl}$ buffer (100 mmol/L, pH 8.0) containing $2 \mathrm{mmol} / \mathrm{L}$ $\mathrm{CaCl}_{2}$ and $5.5 \mathrm{mmol} / \mathrm{L}$ paraoxon (O,O-diethyl-O-pnitrophenylphosphate; Sigma Chemical Co). The rate of generation of p-nitrophenol was determined at
$412 \mathrm{~nm}, 37^{\circ} \mathrm{C}$, with the use of a continuously recording spectrophotometer (Beckman DU-68). Serum glucose levels were measured using a Glucose Oxidase commercial kit (Sigma, St. Louis, Mo, USA).

\subsubsection{Inflammatory markers}

Serum adiponectin (Mercodia, eBioscience) and TNF- $\alpha$ and VEGF (Quantikine ${ }^{\circledR}$, R\&D Systems, Minneapolis, USA) were evaluated by using standard commercial enzyme-linked immunoassays. CRP was evaluated by immunoturbidimetry, using commercially available kits (CRP [latex] High-Sensitivity, Roche Diagnostics).

\subsection{Statistical analysis}

Statistical analysis was performed by using the IBM Statistical Package for Social Sciences (SPSS) for Windows (SPSS, Inc., Chicago IL, version 19.0). The distribution of continuous variables was analyzed using Kolmogorov-Smirnov tests, to assess significant departures from Normality. Comparisons between groups were performed using Fisher's exact test or Mann-Whitney U test. Adjustment of statistical differences for confounding factors was performed using analysis of covariance (ANCOVA). The association between categorical variables was analyzed using Pearson's test. Bonferroni correction was applied to multiple comparisons. Statistical significance was accepted at $\mathrm{P}$ less than 0.05 .

\section{Results}

\subsection{Anthropometric data}

The demographic and anthropometric data of patients and controls are summarized in Table 1. Thirty MS patients were recruited, 7 men and 23 women, 
Table 2

Markers of lipid, inflammatory and angiogenic profile of multiple sclerosis (MS) patients and age and sex-matched controls

\begin{tabular}{|c|c|c|c|}
\hline Parameters & Controls $(n=66)$ & MS patients $(n=30)$ & $P^{*}$ \\
\hline Total-c (mmol/L) & $4.89(4.49-5.14)$ & $4.57(4.32-5.27)$ & 0.403 \\
\hline TGs (mmol/L) & $0.86(0.64-1.18)$ & $1.07(0.88-1.87)$ & 0.000 \\
\hline LDL-c (mmol/L) & $2.96(2.57-3.50)$ & $2.51(2.08-3.09)$ & 0.016 \\
\hline${ }^{\dagger} \mathrm{Ox}-\mathrm{LDL}(\mathrm{U} / \mathrm{L})$ & $28.95(23.91-34.45)$ & $91.63(74.47-116.99)$ & 0.000 \\
\hline Ox-LDL/LDL-c ratio & $10.00(8.08-11.15)$ & $35.38(31.54-43.85)$ & 0.000 \\
\hline HDL-c (mmol/L) & $1.46(1.21-1.83)$ & $1.52(1.31-1.69)$ & 0.854 \\
\hline Non-HDL-c (mmol/L) & $3.33(2.94-3.93)$ & $3.18(2.71-3.79)$ & 0.611 \\
\hline Total-Chol/HDL-c & $3.21(2.73-4.02)$ & $3.19(2.62-3.93)$ & 0.401 \\
\hline LDL-c/HDL-c ratio & $1.96(1.53-2.56)$ & $1.64(1.21-2.35)$ & 0.041 \\
\hline Large HDL (mmol/L) & $1.08(0.76-1.38)$ & $0.85(0.76-1.07)$ & 0.204 \\
\hline Intermediate HDL (mmol/L) & $1.16(0.97-1.34)$ & $1.25(1.16-1.29)$ & 0.086 \\
\hline Small HDL (mmol/L) & $0.40(0.25-0.52)$ & $0.50(0.36-0.57)$ & 0.040 \\
\hline PON1 (nmolpnitrofenol/mL/min) & $423.83(339.38-544.89)$ & $453.08(361.14-566.49)$ & 0.484 \\
\hline PON 1/HDL-c ratio & $281.92(210.77-433.85)$ & $275.77(222.23-379.23)$ & 0.868 \\
\hline PON 1/Large HDL-c & $431.54(296.92-631.92)$ & $512.31(384.23-707.69)$ & 0.179 \\
\hline PON 1/Intermediate HDL-c & $410.77(314.62-528.46)$ & $361.15(295.00-441.92)$ & 0.471 \\
\hline PON 1/Small HDL-c & $1251.92(836.92-1731.92)$ & $1000.00(705.77-1277.69)$ & 0.087 \\
\hline $\mathrm{CRP}(\mu \mathrm{g} / \mathrm{mL})$ & $0.14(0.04-0.52)$ & $0.50(0.50-0.53)$ & 0.000 \\
\hline VEGF (pg/mL) & $232.20(110.70-494.28)$ & $334.82(180.27-402.09)$ & 0.358 \\
\hline $\mathrm{TNF}-\alpha(\mathrm{pg} / \mathrm{mL})$ & $1.11(0.85-1.71)$ & $1.26(0.93-1.76)$ & 0.530 \\
\hline Adiponectin $(\mathrm{ng} / \mathrm{mL})$ & $8.48(4.21-13.59)$ & $12.61(7.91-12.10)$ & 0.023 \\
\hline
\end{tabular}

Results are presented as median (inter quartile range). ${ }^{*}$ Mann-Whitney test. CRP, C-reactive protein; HDL-c, high-density lipoprotein cholesterol; LDL-c, low-density lipoprotein cholesterol; Ox-LDL, oxidized low-density lipoprotein; PON1, paraoxonase 1; TGs, triglycerides; TNF$\alpha$, tumour necrosis factor alpha; Total-c, total cholesterol; VEGF, vascular endothelial growth factor. ${ }^{\dagger} P$ values adjusted for non-HDL-c plus Total-Chol/HDL-c, for non-HDL-c and for Total-Chol/HDL-c: $P<0.001$ for the 3 adjustments.

with a median age of 35.00 years old (inter quartile range: $28.25-40.25$ ). All these patients were clinically evaluated using EDSS at the day of study enrollment, scoring a median value of 2.0 (1.50-3.13), which means, according to the scale, "minimal disability in 1 functional system" (the EDSS scores range from 0 [no disability] to 10 [death]). Patient's body mass index (BMI) had a median value of 23.75 (21.98$28.43) \mathrm{Kg} / \mathrm{m}^{2}$, which is considered in the normal range (18.5 to $25 \mathrm{Kg} / \mathrm{m}^{2}$ - healthy weight) and no statistically significant difference was found considering this aspect in the control group $(P=0.931)$. Similar situation was found regarding waist circumference and fasting glycemia. Patient's systolic (median of $115.00 \mathrm{mmHg}$ [110.00-121.00]) and diastolic (median of $72.50 \mathrm{mmHg}$ [69.00-77.00]) blood pressure values were significantly lower than in the control group $(P=$ 0.011 and $P=0.001$, respectively).

\subsection{Markers of lipid, inflammatory and angiogenic profile}

Table 2 summarizes the results obtained for all the markers of lipid, inflammatory and angiogenic profile which have been tested in the study. In terms of classic lipid profile, a significant difference was observed between patients and controls regarding: 1) the serum contents of triglycerides (TGs), which was higher in MS patients (median of $1.07 \mathrm{mmol} / \mathrm{L}$ [0.881.87], $P<0.001)$; 2) the serum concentrations of LDL cholesterol (LDL-c), lower in MS patients group (median of $2.51 \mathrm{mmol} / \mathrm{L}$ [2.08-3.09], $P=0.016$ ). Despite these differences, particularly regarding patient's triglyceridemia, it must be emphasized that it is within the range considered normal $(<3.9 \mathrm{mmol} / \mathrm{L})$. No statistically significant differences were found between both groups' levels of total cholesterol (Total-c) and HDL-cholesterol (HDL-c) $(P=0.403$ and $P=0.854$, respectively). Only 1 patient was under treatment with a statin (rosuvastatin, $5 \mathrm{mg}$ per day).

When looking for new biochemical markers of CVR, the apparently protective lipid profile described for MS patients changed notoriously: Ox-LDL is significantly higher (median of 91.63 U/L [74.47$116.99])$ in patients $(P<0.001)$, even after adjustment for non-HDL-c and/or Total-Chol/HDL-c ratio (Table 2). Similar change was obtained for the more atherogenic (or at least less protective) small HDL (median of $0.50 \mathrm{mmol} / \mathrm{L}$ [0.36-0.57]; $P=0.040$ ). No differences were found between groups regarding the content of protective large HDL $(P=0.204)$ and intermediate HDL $(P=0.086)$. The increased OxLDL content in MS patients is reflected in a higher Ox-LDL/LDL-c ratio $(P<0.001)$. PON1 activity and 

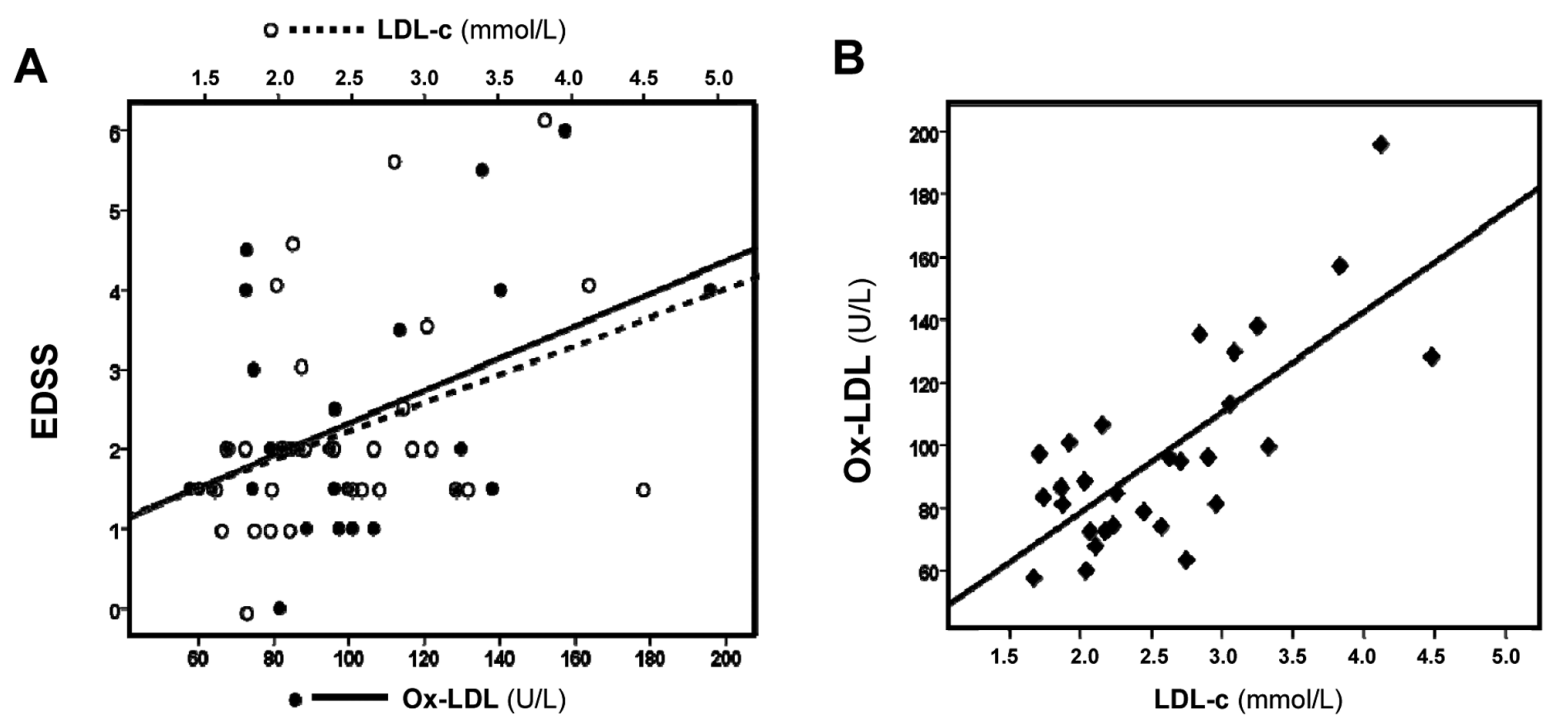

Fig. 1. Correlation of EDSS score with Ox-LDL and LDL-c (A) and of Ox-LDL with LDL-c (B) in multiple sclerosis (MS) patients. (Colours are visible in the online version of the article; http://dx.doi.org/10.3233/DMA-130979)

o ...... LDL-c (mmol/L)

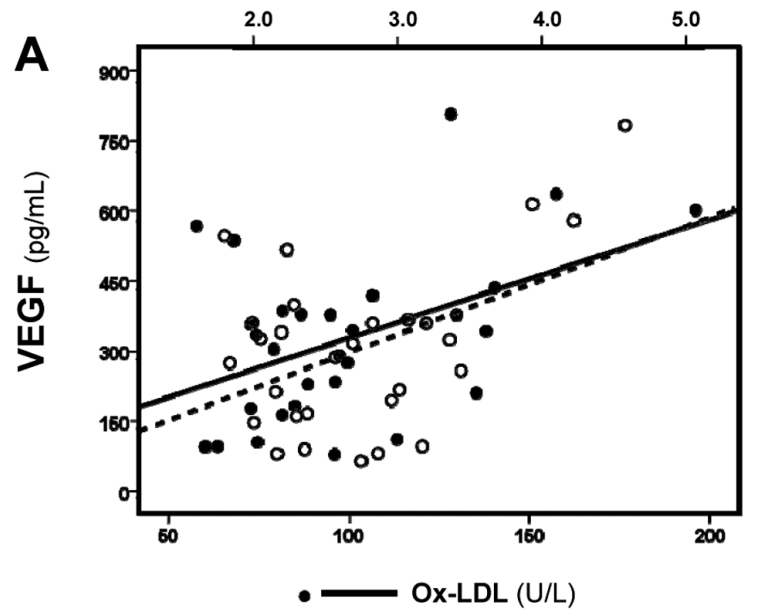

$\nabla \cdot \ldots . .$. Small HDL-c (mmol/L)

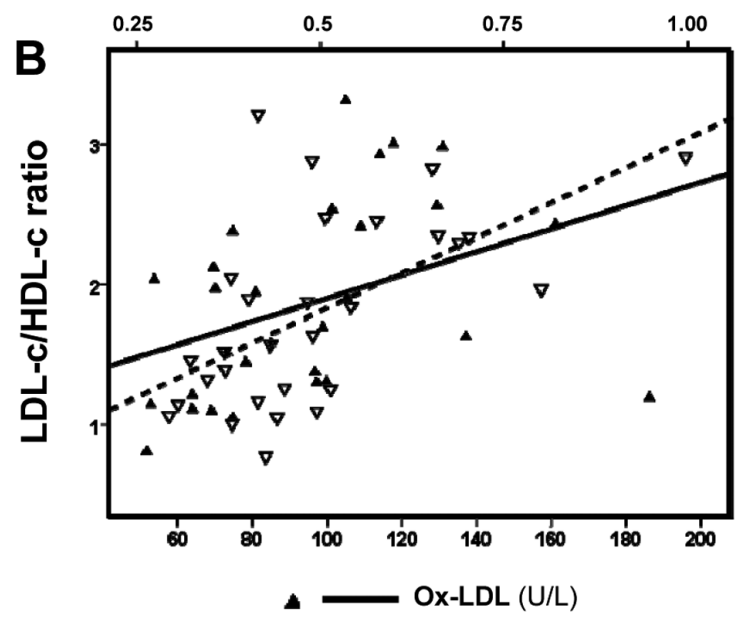

Fig. 2. Correlation of serum VEGF with Ox-LDL and LDL-c (A) and of LDL-c/HDL-c ratio with Ox-LDL and Small HDL subpopulation (B) in multiple sclerosis (MS) patients. (Colours are visible in the online version of the article; http://dx.doi.org/10.3233/DMA-130979)

TNF- $\alpha$ values were unchanged between groups $(P=$ 0.484 and $P=0.530$, respectively). C reactive protein (CRP) levels were higher in MS patients (median of $0.50 \mu \mathrm{g} / \mathrm{mL}$ [0.50-0.53]; $P<0.001$ ), which seems to be balanced by an increased amount of adiponectin, an anti-inflammatory molecule, detected in the same group (median of $12.61 \mathrm{ng} / \mathrm{mL}$ [7.91-12.10]; $P=$ 0.023). Despite not reaching statistical significance, VEGF levels tend to be higher $(>44 \%)$ in MS patients than in control population.

\subsection{Ox-LDL is correlated with EDSS, VEGF, small $H D L$ and triglycerides}

In a correlation analysis, establishing a bridge between laboratorial determinations and patients clinical stage, as measured by the EDSS, a positive and significant correlation was found between EDSS score and Ox-LDL $(r=0.458 ; P=0.011)$ and LDL-c $(r=$ 0.366; $P=0.050$ ) serum levels (Fig. 1(A)). Similar correlation was also found between LDL-c and OxLDL contents ( $r=0.742 ; P<0.001)$ (Fig. 1(B)). 
Serum VEGF levels were also positively and significantly correlated with Ox-LDL $(r=0.453 ; P=$ $0.014)$ and LDL-c contents $(r=0.459 ; P=0.014)$ (Fig. 2(A)). A similar relationship was established between LDL-c/HDL-c ratio and small HDL-c $(r=$ $0.388 ; P=0.037)$ and Ox-LDL $(r=0.582 ; P=$ 0.001) (Fig. 2(B)).

Other established correlations, in MS patients group, were the following: 1$) \mathrm{Ox}-\mathrm{LDL}$ and TGs $(r=0.456 ; P$ $=0.011) ; 2)$ EDSS and total-c $(r=0.404 ; P=0.027)$;

$3)$ LDL-c and glycemia ( $r=0.453 ; P=0.014)$; 4) a negative and statistically significant correlation was defined between small HDL-c and adiponectin ( $r=$ $-0.359 ; P=0.050)$.

\section{Discussion}

In this paper, we have reported results indicating that MS patients could have an increased CVR, based on elevation of some aggressive and atherogenic molecules in serum, particularly Ox-LDL and small HDL-c. However, this risk profile is hidden behind an apparently normal classic lipid panel (despite the significant difference observed in terms of serum TGs contents, but within the normal range), reinforcing the importance of looking for some new biochemical markers of CVR in MS patients. In our study population, it was also possible to correlate those new lipid profile abnormalities with clinical staging, evaluated by the usage of the universal EDSS score, which raises the possibility of such aggressive molecules to be involved in the process of disease progression.

It is well known that cholesterol is an important component of myelin. Lipids, especially lipoproteins, are involved in the regulation of neural functions in the CNS through local mechanisms that are linked to systemic lipid metabolism [19,20]. Under normal and physiological conditions, high concentrations of HDL and LDL are present in CNS, as a result of transport across the brain-blood barrier (BBB) [21]. However, dyslipidemia can potentiate inflammatory processes at the vascular endothelium, lead to the production of adhesion molecules and to the recruitment of leukocytes [22,23]. The recruitment and extravasation of immune cells across the activated endothelium of the BBB is considered to be a critical step in MS pathogenesis. A significant amount of cerebral vascular endothelial dysfunction has been reported in MS [24], accompanied by some degree of cerebral hypoperfusion [25]. Weinstock-Guttman et al., studying a population of 492 MS patients, concluded that serum lipid profile had modest effects on disease progression. Worsening disability was associated with higher levels of LDL, total cholesterol and triglycerides, whereas higher levels of HDL were associated with minor levels of acute inflammatory activity, in magnetic resonance imaging (MRI) [11]. However (and even taking into account that our study did not focus on imagiological analysis), our data draw attention to the importance of measuring new markers of dyslipidemia (particularly Ox-LDL and small HDL), more reliably related with the disease and its progression, continuing to support the working hypothesis of thinking that the proinflammatory and thrombogenic mechanisms associated with an abnormal lipid profile could contribute to MS progression, through diverse processes at the BBB vascular endothelium. The relevance of "non-classic" markers of cardiovascular risk, such as oxidized LDL, has been increasingly demonstrated in young or adult subjects with coronary artery disease [26,27].

An important limitation of our study is sample size (only 30 patients were included and analyzed). Still, only 1 patient was under treatment with a statin, excluding the possible and meaningful interference of this kind of drugs in our results. Some studies of statin treatment in MS were performed, but they originated mixed results [28-33]. In a study of 30 MS patients, the treatment with simvastatin resulted in a significant reduction in the number and volume of contrastenhancing lesions, on serial monthly MRI [28]. A posthoc analysis of the interferon beta treated control arm of the SENTINEL study did not show any effect of statins on the following endpoints: adjusted annualized relapse rate, disability progression, number of contrastenhancing lesions and number of new or enlarging T2hyperintense lesions over 2 years [31]. The STAyCIS study tried to assess the effect of statin treatment in slowing the conversion of clinical isolated syndromes to clinically definite MS, but it did not meet its primary endpoint (development of 3 or more new T2 lesions or 1 clinical relapse within 12 months) [32]. The SIMCOMBIN trial indicated that statin treatment did not provide benefit in MS patients on interferon beta [33]. According to our knowledge, this is the first study showing a strong correlation between EDSS score and Ox-LDL and LDL, suggesting a negative impact of LDL-c, and particularly of Ox-LDL, on EDSS. Those markers of early CVR may raise special attention for premature intervention, in order to prevent cardiovascular disease in MS patients. Lifestyle changes, including adoption of a healthier diet and regular ex- 
ercise, providing improvement on serum lipid profile, may be beneficial for MS patients to improve their neurological condition. Early introduction of an antidyslipidemic drug, such as a statin, can be tested in the future, looking for an impact on new markers of cardiometabolic risk, such as those described in this paper.

It must be emphasized that these are young patients, in no way resembling the typical profile of atherosclerotic individuals seen in clinical practice - MS patients are not obese, they do not suffer from hypertension or diabetes and, if we only consider the classical lipid panel, they present normal-range values. The striking age and sex-matching between patients and control volunteers should be also noted. When compared with this control subjects, the young MS patients show an early unfavorable vascular risk profile, traduced in the presence of more aggressive and atherogenic blood particles, such as ox-LDL and small HDL. Patient's angiogenic function also contributes for this risk raising mixture. VEGF may stimulate the formation of microvessels inside the atherosclerotic plaque (which may become disrupted and cause intraplaque hemorrhage), has a role on thrombus formation and significantly contributes for global cardiometabolic risk. We detected an elevation of patients' VEGF levels, of about $44 \%$, comparing to controls. This result didn't reach statistical significance, but also contributes to draw attention to this molecule in MS patients in futures studies. Chronic inflammation is also an important player in raising vascular risk. In a study published in 2005, Soilu-Hänninen et al. verified that CRP levels, measured by a high-sensitivity technique, were similar in patients with MS and healthy controls, but higher during MS relapses than in remission $(p=0.010)$ [34]. They also verified that patients under treatment with interferon beta-1a had lower levels of serum CRP, comparing with those treated with placebo in the PRISMS (Prevention of Relapses and Disability by Interferon beta-1a Subcutaneously in Multiple Sclerosis) study $(p=0.035)$. All patients included in our study were under treatment with disease modifying drugs (first line therapy) or natalizumab. Serum CRP levels were within the normal range for all of them, although values were higher (reaching statistical significance) than those found in healthy volunteers, reinforcing the concept of chronic inflammation being present in this clinical situation. This seemed to be balanced by a similar variation in the levels of adiponectin, which normally plays an anti-inflammatory role, as previously said. But this dynamic relationship deserves further investigation, in order to understand how these molecules interact and contribute for the global CVR in MS.

\section{Conclusions}

MS patients seem to present a profile indicative of an increased CVR, when compared with healthy subjects of well matched age and sex. The clinical activity of the disease (given by the EDSS score) was directly and significantly correlated with some markers of CVR, particularly Ox-LDL content, among other putatively good new markers that deserve further attention (small HDL subpopulation and VEGF). The early atherogenic risk found in this population should receive special attention and, in the future, after better understand the mechanisms involved and after better characterize the molecular players interacting, this issue might be enough to recommend early pharmacotherapeutic intervention to prevent premature development of cardiovascular disease in these patients.

\section{Acknowledgments}

This study was supported by the Portuguese Group for the Study of Multiple Sclerosis, through a grant received from Merck Serono.

\section{Conflict of interest}

The authors report no conflict of interest.

\section{References}

[1] World Health Organization: Atlas Multiple Sclerosis Resources in the World. Geneva, WHO 2008, http://www.who. int/mental_health/neurology/Atlas_MS_WEB.pdf (accessed on 20 July 2012).

[2] Ramagopalan SV, Dobson R, Meier UC, Giovannoni G. Multiple sclerosis: Risk factors, prodromes, and potential causal pathways. Lancet Neurol. 2010;9(7):727-39.

[3] Acevedo AR, Nava C, Arriada N, Violante A, Corona T. Cardiovascular dysfunction in multiple sclerosis. Acta Neurol Scand. 2000;101(2):85-8.

[4] Christiansen CF, Christensen S, Farkas DK, Miret M, Sørensen HT, Pedersen L. Risk of arterial cardiovascular diseases in patients with multiple sclerosis: A population-based cohort study. Neuroepidemiology. 2010;35(4):267-74.

[5] Ranadive SM, Yan H, Weikert M, Lane AD, Linden MA, Baynard T, Motl RW, Fernhall B. Vascular dysfunction and physical activity in multiple sclerosis. Med Sci Sports Exerc. 2012;44(2):238-43.

[6] Merkelbach S, Haensch CA, Hemmer B, Koehler J, König $\mathrm{NH}$, Ziemssen T. Multiple sclerosis and the autonomic nervous system. J Neurol. 2006;253 Suppl 1:I21-5. 
[7] Sanya EO, Tutaj M, Brown CM, Goel N, Neundörfer B, Hilz MJ. Abnormal heart rate and blood pressure responses to baroreflex stimulation in multiple sclerosis patients. Clin $\mathrm{Au}-$ ton Res. 2005;15(3):213-8.

[8] Olindo S, Guillon B, Helias J, Phillibert B, Magne C, Fève JR. Decrease in heart ventricular ejection fraction during multiple sclerosis. Eur J Neurol. 2002;9(3):287-91.

[9] Kanabrocki EL, Ryan MD, Hermida RC, Ayala DE, Scott GS, Murray D, Bremner WF, Third JL, Johnson MC, Foley S, Van Cauteren J, Shah F, Shirazi P, Nemchausky BA, Hooper DC. Altered circadian relationship between serum nitric oxide, carbon dioxide, and uric acid in multiple sclerosis. Chronobiol Int. 2004;21(4-5):739-58.

[10] Mirshafiey A, Jadidi-Niaragh F. Prostaglandins in pathogenesis and treatment of multiple sclerosis. Immunopharmacol Immunotoxicol. 2010;32(4):543-54.

[11] Weinstock-Guttman B, Zivadinov R, Mahfooz N, Carl E, Drake A, Schneider J, Teter B, Hussein S, Mehta B, Weiskopf M, Durfee J, Bergsland N, Ramanathan M. Serum lipid profiles are associated with disability and MRI outcomes in multiple sclerosis. J Neuroinflammation. 2011;4(8):127.

[12] Mackness MI, Durrington PN, Mackness B. The role of paraoxonase 1 activity in cardiovascular disease: Potential for therapeutic intervention. Am J Cardiovasc Drugs. 2004;4(4): 211-217.

[13] Mangge H, Almer G, Truschnig-Wilders M, Schmidt A, Gasser R, Fuchs D. Inflammation, adiponectin, obesity and cardiovascular risk. Curr Med Chem. 2010;17(36):4511-20.

[14] Huang SS, Huang PH, Chen YH, Chiang KH, Chen JW, Lin SJ. Association of adiponectin with future cardiovascular events in patients after acute myocardial infarction. J Atheroscler Thromb. 2010;17(3):295-303

[15] Girardi F, Franceschi E, Brandes AA. Cardiovascular safety of VEGF-targeting therapies: current evidence and handling strategies. Oncologist. 2010;15(7):683-94.

[16] Iacobaeus E, Amoudruz P, Ström M, Khademi M, Brundin L, Hillert J, Kockum I, Malmström V, Olsson T, Tham E, Piehl F. The expression of VEGF-A is down regulated in peripheral blood mononuclear cells of patients with secondary progressive multiple sclerosis. PLoS One. 2011;6(5):e19138.

[17] Kurtzke JF. Rating neurological impairment in multiple sclerosis: An expanded disability status scale (EDSS). Neurology. 1983;33(11):1444-1452.

[18] Polman CH, Reingold SC, Banwell B, Clanet M, Cohen JA, Filippi M, Fujihara K, Havrdova E, Hutchinson M, Kappos L, Lublin FD, Montalban X, O'Connor P, Sandberg-Wollheim M, Thompson AJ, Waubant E, Weinshenker B, Wolinsky JS. Diagnostic criteria for multiple sclerosis: 2010 revisions to the McDonald criteria. Ann Neurol. 2011;69(2):292-302.

[19] Gotthardt M, Trommsdorff M, Nevitt MF, Shelton J, Richardson JA, Stockinger W, Nimpf J, Herz J. Interactions of the low density lipoprotein receptor gene family with cytosolic adaptor and scaffold proteins suggest diverse biological functions in cellular communication and signal transduction. J Biol Chem. 2000;275(33):25616-24.

[20] Stockinger W, Brandes C, Fasching D, Hermann M, Gotthardt M, Herz J, Schneider WJ, Nimpf J. The reelin receptor ApoER2 recruits JNK-interacting proteins-1 and -2. J Biol Chem. 2000;275(33):25625-32.

[21] Borghini I, Barja F, Pometta D, James RW. Characterization of subpopulations of lipoprotein particles isolated from human cerebrospinal fluid. Biochem Biophys Acta. 1995; 1255(2):192-200.

[22] Cybulsky MI, Jr. Gimbrone MA. Endothelial expression of a mononuclear leukocyte adhesion molecule during atherogen esis. Science. 1991; 251(4995):788-791.

[23] Stokes KY, Calahan L, Hamric CM, Russell JM, Granger DN. CD40/CD40L contributes to hypercholesterolemia-induced microvascular inflammation. Am J Physiol Heart Circ Physiol. 2009;296(3):H689-97.

[24] Minagar A, Jy W, Jimenez JJ, Alexander JS. Multiple sclerosis as a vascular disease. Neurol Res. 2006;28(3):230-5.

[25] De Keyser J, Steen C, Mostert JP, Koch MW. Hypoperfusion of the cerebral white matter in multiple sclerosis: Possible mechanisms and pathophysiological significance. J Cereb Blood Flow Metab. 2008;28(10):1645-51.

[26] Huang H, Ma R, Liu D, Liu C, Ma Y, Mai W, Dong Y. Oxidized low-density lipoprotein cholesterol and the ratio in the diagnosis and evaluation of therapeutic effect in patients with coronary artery disease. Dis Markers. 2012 Oct 22. [Epub ahead of print].

[27] Huang Y, Hu Y, Mai W, Cai X, Song Y, Wu Y, Dong Y, Huang H, He Z, Li W, Yang Y, Rao S. Plasma oxidized low-density lipoprotein is an independent risk factor in young patients with coronary artery disease. Dis Markers. 2011;31(5):295301.

[28] Vollmer T, Key L, Durkalski V, Tyor W, Corboy J, MarkovicPlese S, Preiningerova J, Rizzo M, Singh I. Oral simvastatin treatment in relapsing-remitting multiple sclerosis. Lancet. 2004;363(9421):1607-8.

[29] Markovic-Plese S, Jewells V, Speer D. Combining beta interferon and atorvastatin may increase disease activity in multiple sclerosis. Neurology. 2009;72(22):1965-6.

[30] Sellner J, Weber MS, Vollmar P, Mattle HP, Hemmer B, Stüve O. The combination of interferon-beta and HMG-CoA reductase inhibition in multiple sclerosis: Enthusiasm lost too soon? CNS Neurosci Ther. 2010;16(6):362-73.

[31] Rudick RA, Pace A, Rani MR, Hyde R, Panzara M, Appachi S, Shrock J, Maurer SL, Calabresi PA, Confavreux C, Galetta SL, Lublin FD, Radue EW, Ransohoff RM. Effect of statins on clinical and molecular responses to intramuscular interferon beta-1a. Neurology. 2009;72(23):1989-93.

[32] Waubant E, Pelletier D, Mass M, Cohen JA, Kita M, Cross A, Bar-Or A, Vollmer T, Racke M, Stüve O, Schwid S, Goodman A, Kachuck N, Preiningerova J, Weinstock-Guttman B, Calabresi PA, Miller A, Mokhtarani M, Iklé D, Murphy S, Kopetskie H, Ding L, Rosenberg E, Spencer C, Zamvil SS. ITN STAyCIS Study Group; ITN020AI Study Management Team. Randomized controlled trial of atorvastatin in clinically isolated syndrome: The STAyCIS study. Neurology. 2012; 78(15):1171-8

[33] Sorensen PS, Lycke J, Erälinna JP, Edland A, Wu X, Frederiksen JL, Oturai A, Malmeström C, Stenager E, Sellebjerg F, Sondergaard HB. SIMCOMBIN study investigators. Simvastatin as add-on therapy to interferon $\beta$-1a for relapsing-remitting multiple sclerosis (SIMCOMBIN study): A placebo-controlled randomised phase 4 trial. Lancet Neurol. 2011;10(8):691-701.

[34] Soilu-Hänninen M, Koskinen JO, Laaksonen M, Hänninen A, Lilius EM, Waris M. High sensitivity measurement of $\mathrm{CRP}$ and disease progression in multiple sclerosis. Neurology. 2005;65(1):153-5. 


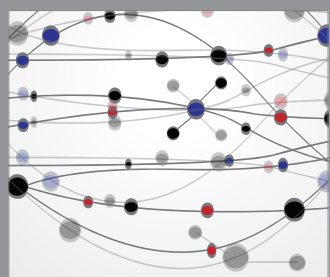

The Scientific World Journal
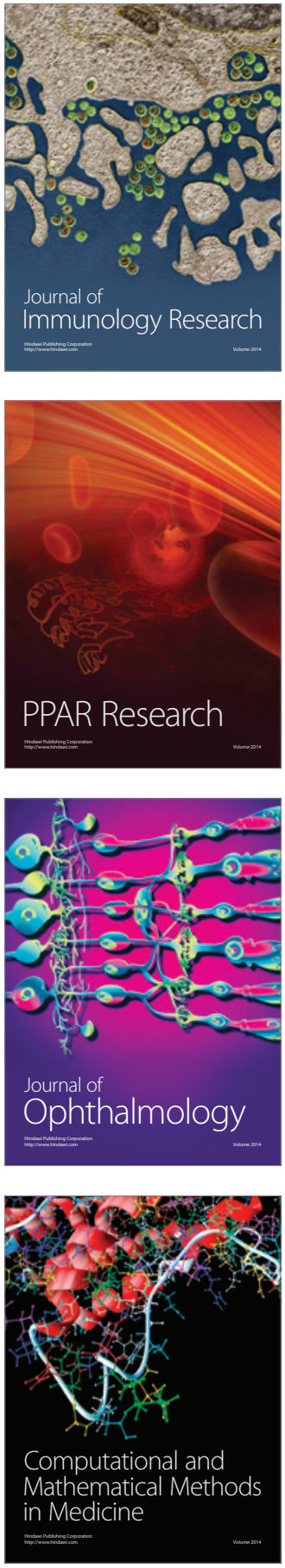

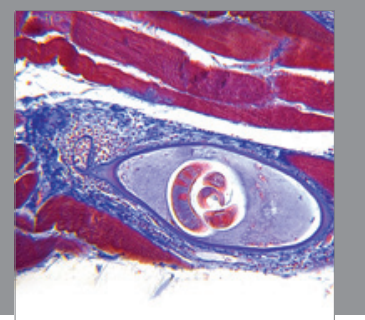

Gastroenterology

Research and Practice
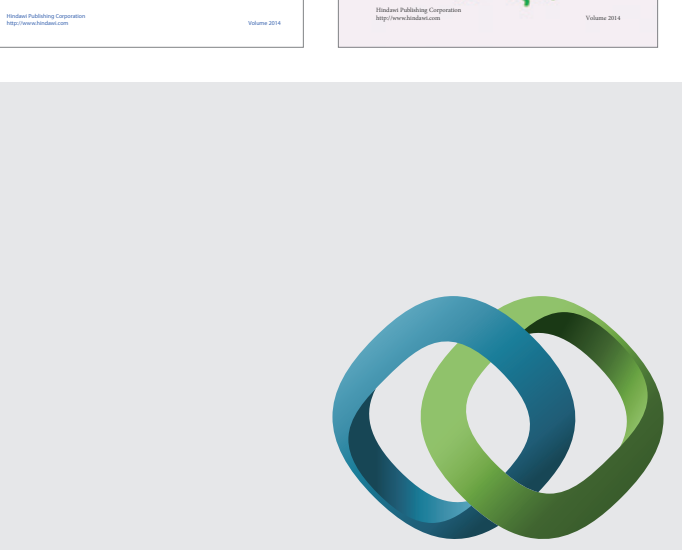

\section{Hindawi}

Submit your manuscripts at

http://www.hindawi.com
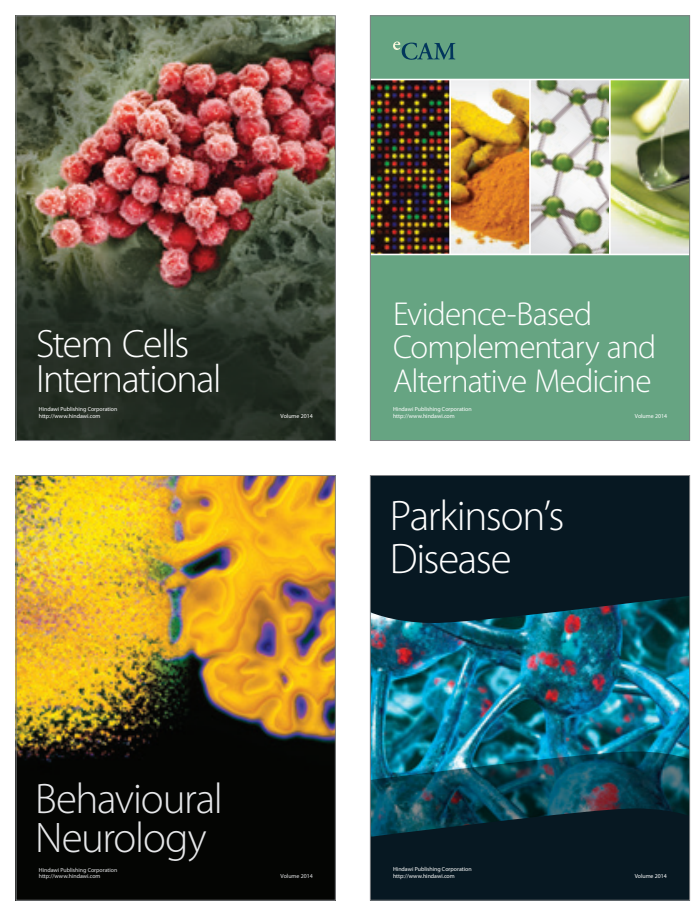

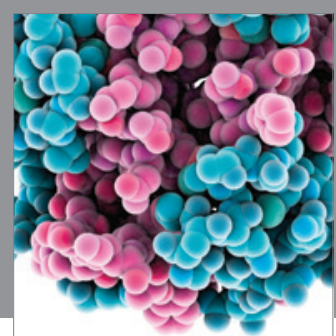

Journal of
Diabetes Research

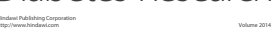

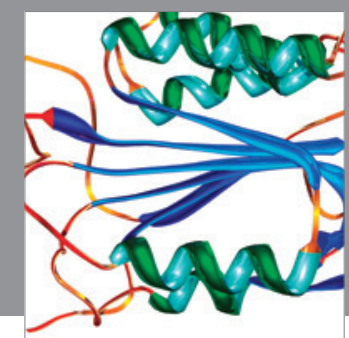

Disease Markers
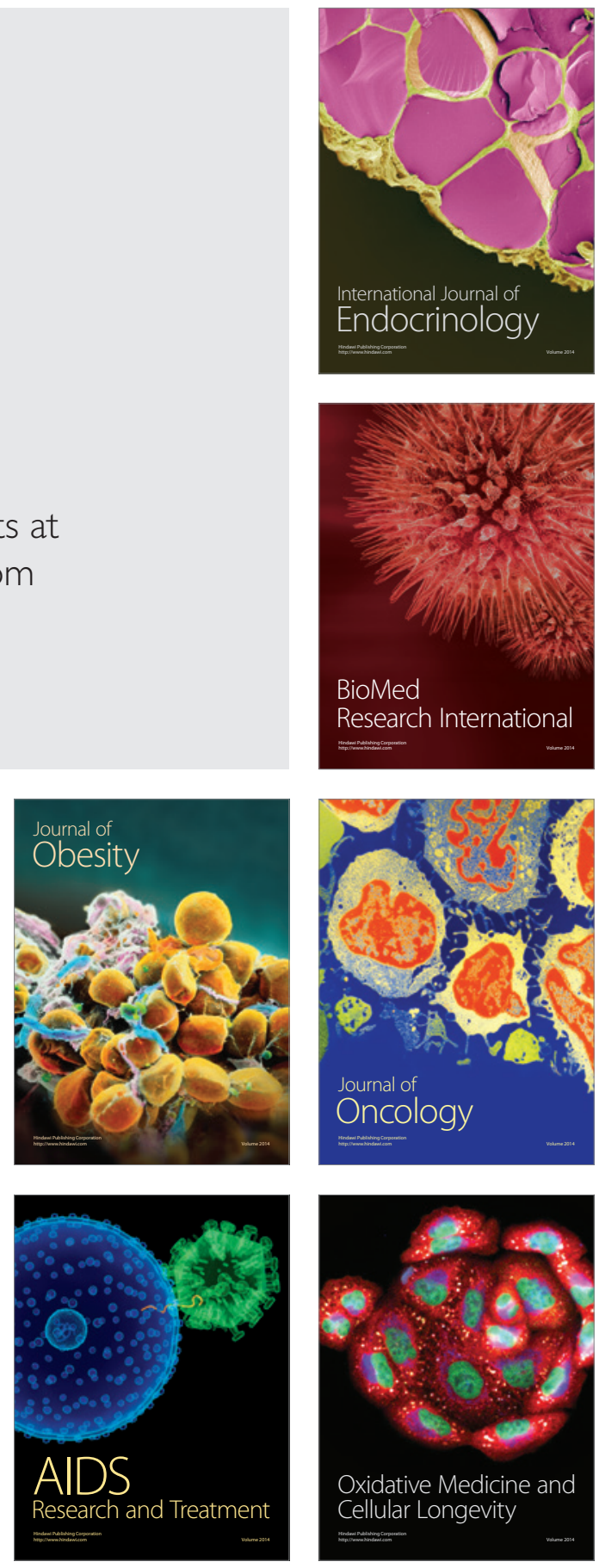